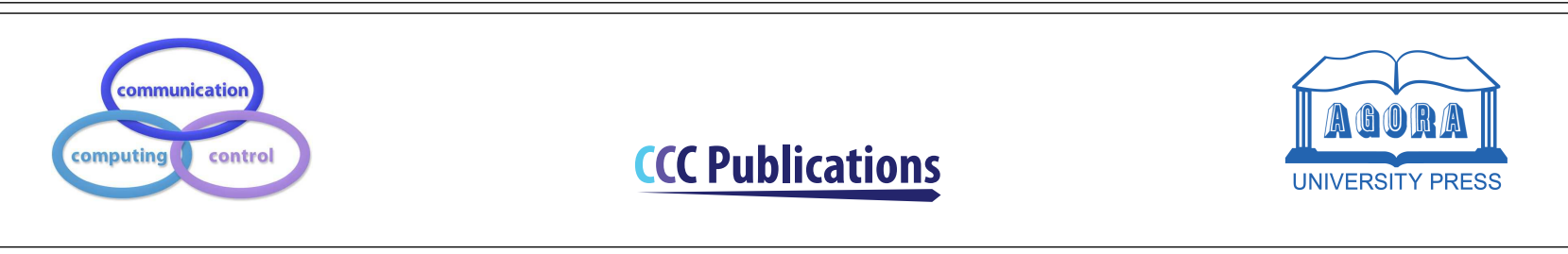

\title{
Technical Solution for a Real-Time Air Quality Monitoring System
}

\author{
A. Simo, S. Dzitac, F. M. Frigura-Iliasa, S. Musuroi, P. Andea, D. Meianu
}

\section{Attila Simo*}

1. Politehnica University Timisoara

300006 Timisoara, Piata Victoriei, 2, Romania

2. Romanian Academy of Scientists

300220 Bucuresti, Ilfov, 3, Romania

*Corresponding author: attila.simo@upt.ro

\section{Simona Dzitac, Dragoș Meianu}

University of Oradea

410087 Oradea, Universitatii, 1, Romania

simona@dzitac.ro,dmeianu@uoradea.ro

\section{Flaviu Mihai Frigura-Iliasa,}

\section{Sorin Musuroi, Petru Andea}

1. Politehnica University Timisoara

300006 Timsoara, Piata Victoriei, 2, Romania

2. Romanian Academy of Scientists

300220 Bucuresti, Ilfov, 3, Romania

flaviu.frigura@upt.ro,

sorin.musuroi@upt.ro, petru.andea@upt.ro

\begin{abstract}
This article will present a simple technical solution for a low-power and real-time air quality monitoring system. The whole package of software and hardware technical solutions applied for recording, transmitting and analyzing data is briefly described. This original monitoring system integrates a single chip microcon-troller, several dedicated air pollution surveillance sensors (for PM10, PM2.5, SO2, NO2, CO, O3, VOC, CO2), a LoRaWAN communication module and an online platform. This system was tested and applied under real field conditions. Depending on the measured values, it provides alerts, or, it can lead to the re-placement of specific components in the exhaust equipment. This article will pre-sent some experimental results, validated also by official measurements of government operated air quality stations.
\end{abstract}

Keywords: wireless sensors network, air quality monitoring, LoRaWAN. 


\section{Introduction}

Nowadays, air quality monitoring in the urban environment, generally, and near the industrial plants, particularly, is becoming an increasingly important issue. Many, today existing air quality monitoring systems are using Wireless Sensor Networks (WSN), Vehicular Sensor Networks (VSN) or other Internet of Things (IoT) based technologies. The shortcomings of these systems, if the technology is not well chosen, (e.g. a system based on ZigBee, NB-IoT, LTE-M) is that they require a large number of sensors and gateways to establish a multi-hop network for ferrying the data to the backend system. Such approach generally increases the network cost and the energy consumption.

If application scenario needs small packages of data, the measuring points has no electricity supply and needs coverage over large geographical area, for such situa-tions, the Low Power Wide Area (LPWA) networks provide the solution.

LPWA networks permits a long communication range, low power consumption, low cost and direct M2M access. There are a number of competing standards in the LPWA network space, such: Sigfox, Dash7, Telensa, NB-IoT, Nwave, LoRa, etc.

This paper presents a part of a more complex work from the LPWA networks field, ongoing in the Power Systems Department from Politehnica University of Timisoara, in collaboration with ETA2U Company. A software-hardware complex of technical solutions for collecting, transmitting and analyzing data is presented within this paper. The proposed pilot system integrates a single chip microcontroller, several calibrated air pollution low-cost monitoring sensors (PM10, PM2.5, SO2, NO2, $\mathrm{CO}, \mathrm{O} 3, \mathrm{VOC}, \mathrm{CO} 2)$, LoRaWAN communication module and an online application. The proposed solution is tested and validated under real field conditions.

Depending on the measured values, the application can generate alerts and can propose the replacement of certain components in the filtration systems, in case of industrial plants. The authors have also dealt with the air pollution-monitoring problem in the past. In [10] they offer a practical model of an automated environment parameters monitoring system for industrial plants, a multifunctional softhardware complex of technical solutions for collecting, transmitting, processing and analyzing data. The proposed system in [10] can embed different type of sensors for chemical emissions monitoring in industrial plants. The whole system is based on the LoRaWAN communication protocol and a self-flying drone system. The current project was born to have real-time data about air parameters, over large geographical area, which helps us manage activities in the territories impelled by devices (e.g. traffic management, construction areas, industrial plants).

In literature, there are many theoretical studies and proposed solutions regarding air pollution monitoring [2], [3, 4, 7, 9] . Many uses cases refer to small geographical areas where WIFI technology is also suitable. Few projects target large geographical areas and require devices to have low energy consumption [6, 11]. Existing air quality monitoring systems are using a specific range of sensors to measure various air pollutants but tend to only have one PM sensor and do not use LPWA networks $[1,8,12]$.

The deployment of a thick, reliable wide area network of air quality monitoring system would improve the ability to identify sources of pollution, understand person-al exposure, complement existing monitoring networks and raise air quality awareness among the population.

\section{Monitoring system description}

Deploying wireless sensors network over large geographical area requires supporting infrastructure. The pilot project where the monitoring devices were to be installed referred to an industrial area of about $25 \mathrm{~km} 2$. The longterm strategy intents that this air quality monitoring device to become part of a smart city infrastructure providing concrete data about the air parameters for local residents.

The main purpose was to create a low-cost device, which, if needed, to be accessible, even for the residents, but, at the same time, to provide correct and indisputable results. A Raspberry Pi Hardware Attached on Top (HAT) provides the computation. In the first stage, the team tried the measurement variant presented in [10], with Waspmote Pro 1.5 and Waspmote 3.0, to which the sensors were added.

Later, this support was replaced with an own data acquisition board, on which the sensors and 
the communication module were integrated.

In this pilot project, we used the LoRaWAN communication protocol, due to its flexibility, ease of use, and other advantages offered versus other LPWA technologies, and to the local coverage.

This project strong point was the fact that the ETA2U Company has a LoRa net-work at the level of the Timisoara municipality, named LoRaTIM. It is a complex network, consisting of Cisco IXM-LPWA-800-16-K9 and Kerlink gateways, with a very good coverage that can be seen on figure 1.

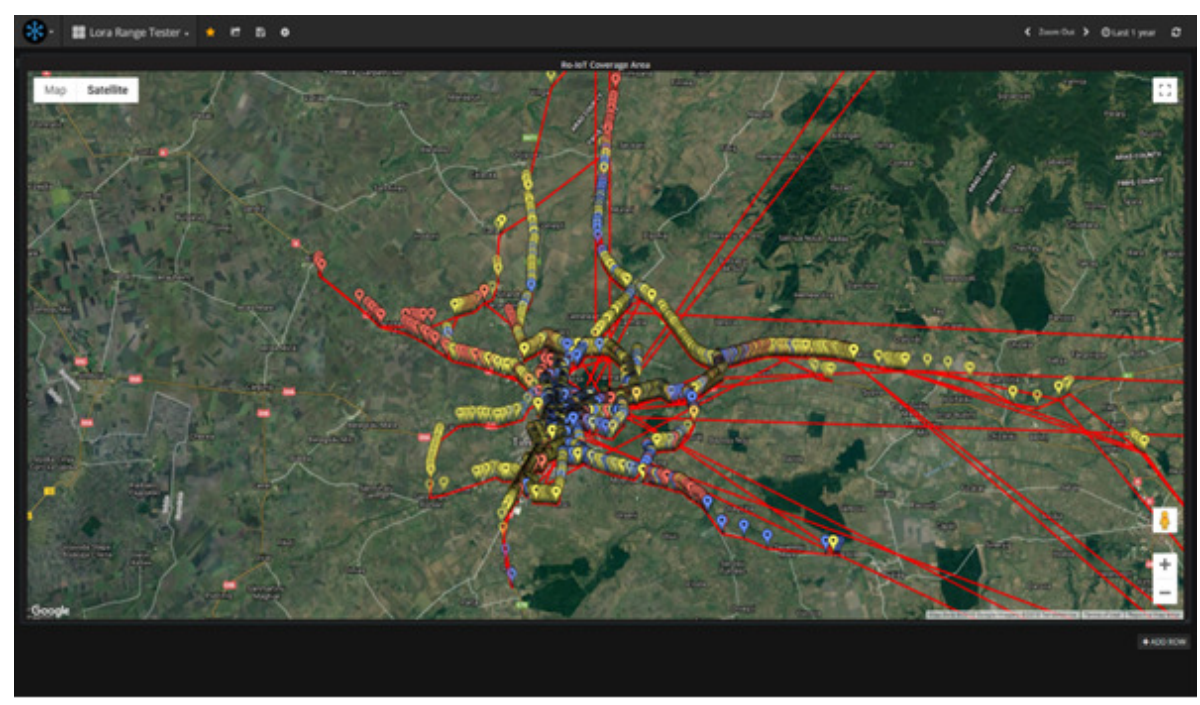

Figure 1: LoRaTIM network coverage

\subsection{Hardware system}

Within this pilot project, the team have planned the construction of six devices, for monitoring air quality issues in an industrial area of Timisoara, Romania. With these six devices, the area of interest is covered. This area being geographically large for data collection, is is necessary to use the LoRaWAN communication protocol.

In the first phase, the solution was tested with the device previously conceived by our team [10], presented in figure 2 .

Some of the results obtained with this version 1 (with calibrated sensors) were very good (partially presented in figure 2), but a major disadvantage was that not all the sensors could be connected on the same sensor board (PM10, PM2.5, SO2, NO2, CO, O3, VOC, CO2). The results for NO2 for June 2018 are presented in figure 3. Similar results were obtained for the other polluting agents, too.

In order to cover the entire range of pollutants that have been proposed, should have had to double the number of sensor boards (figure 2, on the right side), which increased the device cost, the volume and not least the energy consumption. In addition to the sensors that were placed directly on the sensor board, the particulate mat-ter (PM) sensor had to be connected to this. This further complicates the situation.

In this pilot project, the team used the HPMA115S0-XXX Honeywell particulate matter sensor. This is a laser-based sensor which detects and counts particles using light scattering [5].

The detection concentration range is $0 \mu \mathrm{g} / \mathrm{m} 3$ to $1,000 \mu \mathrm{g} / \mathrm{m} 3$, it is fully calibrated, with $<6 \mathrm{~s}$ response time and max $80 \mathrm{~mA}$ current supply. Based on IEC61000 stable operation, plus/minus 15\% accuracy (PM2.5, PM10) it can be considered a sensor that meets the needs. Because of these, the team decided to design an own sensor board (one board per device), on which can be connected all the sensors (SO2, NO2, CO, O3, VOC, CO2), HPMA115S0-XXX, the LoRaWAN HAT and Raspberry Pi HAT (as shown in figure 4). Thus, on this board can be connected all the seven sensors plus the rest of the device elements.

All components, are enclosed in an plastic enclosure with IP66 IP rating (as in figure 9), having $360 \mathrm{~mm} \times 270 \mathrm{~mm} \times 165 \mathrm{~mm}(\mathrm{H} \times \mathrm{W} \times \mathrm{D})$ dimensions. Two grids $(6 \times 4)$ of $10 \mathrm{~mm}$ holes were drilled 


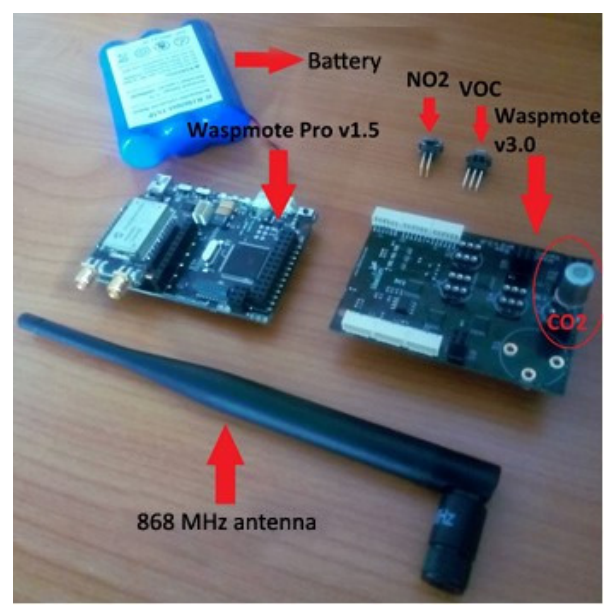

Figure 2: Hardware components

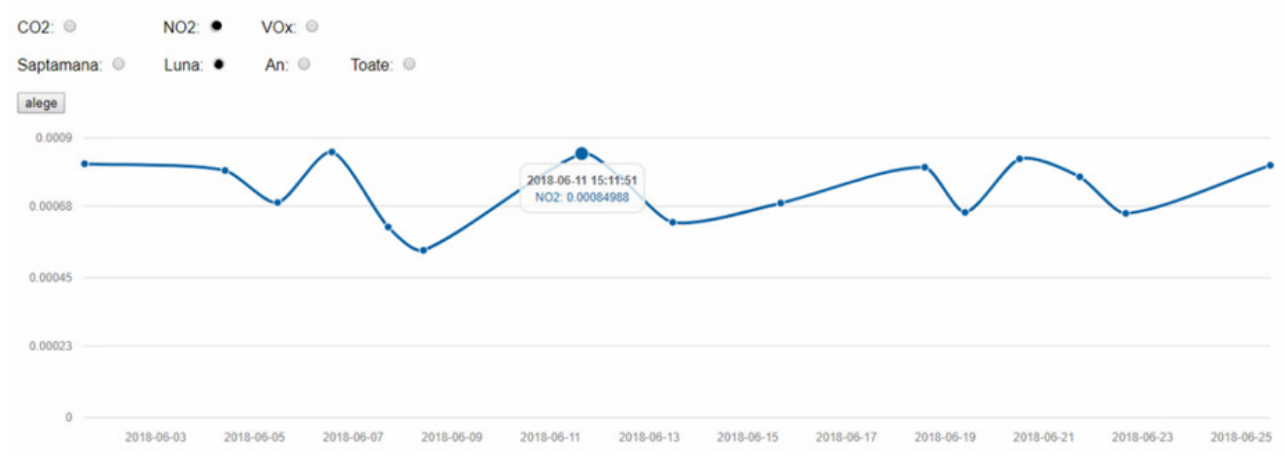

Figure 3: Values recorded for NO2 for one month

on both sides of the enclosure to ensure a constant air flow, for SO2, NO2, CO, O3, VOC and CO2 sensors. The HPMA115S0-XXX PM sensor was taken out of the box.

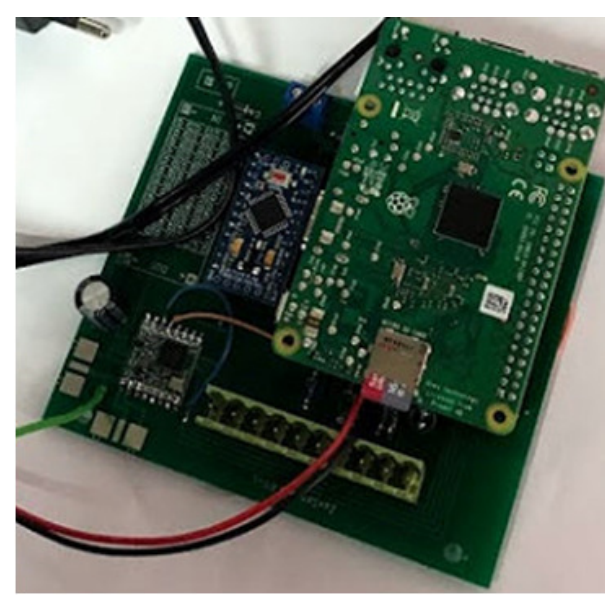

Figure 4: Own data acquisition board, developed by ETA2U Innovation

\subsection{Software system}

In parallel with the development of the monitoring devices, an online platform has been designed for data analytics and different alerts setting.

On this platform, the user can select from the list or on the map the industrial platform of interest, after which he can select the device from which he wants the information about air quality parameters. Figure 5 shows the design of the platform. Because the administrators of the industrial platform did 
Table 1: List of hardware used to build one monitoring device

\begin{tabular}{|l|l|l|}
\hline Item & Quantity & Unit Price (Euro) \\
\hline Raspberry Pi HAT & 1 & 40 \\
\hline Gas sensors & 7 & 10 \\
\hline Honeywell PM Sensor & 1 & 35 \\
\hline LoRaWAN HAT + antenna & 1 & 45 \\
\hline Plastic enclosure & 1 & 37 \\
\hline
\end{tabular}

not agree, the location of the devices could not be displayed on the map.

The online platform was developed using HTML, CSS, PHP, Javascript and SQL.

The platform offers us the possibility of tracking the evolution of air parameters, in the form of graphs. Data can be displayed per day, per week, per month for each parameter separately (figure 5) or all on the same graph. Because the pilot project is dedicated to local use, the language used in the platform is the local language (Romanian). In the future will be added the option by which users can choose another international language.

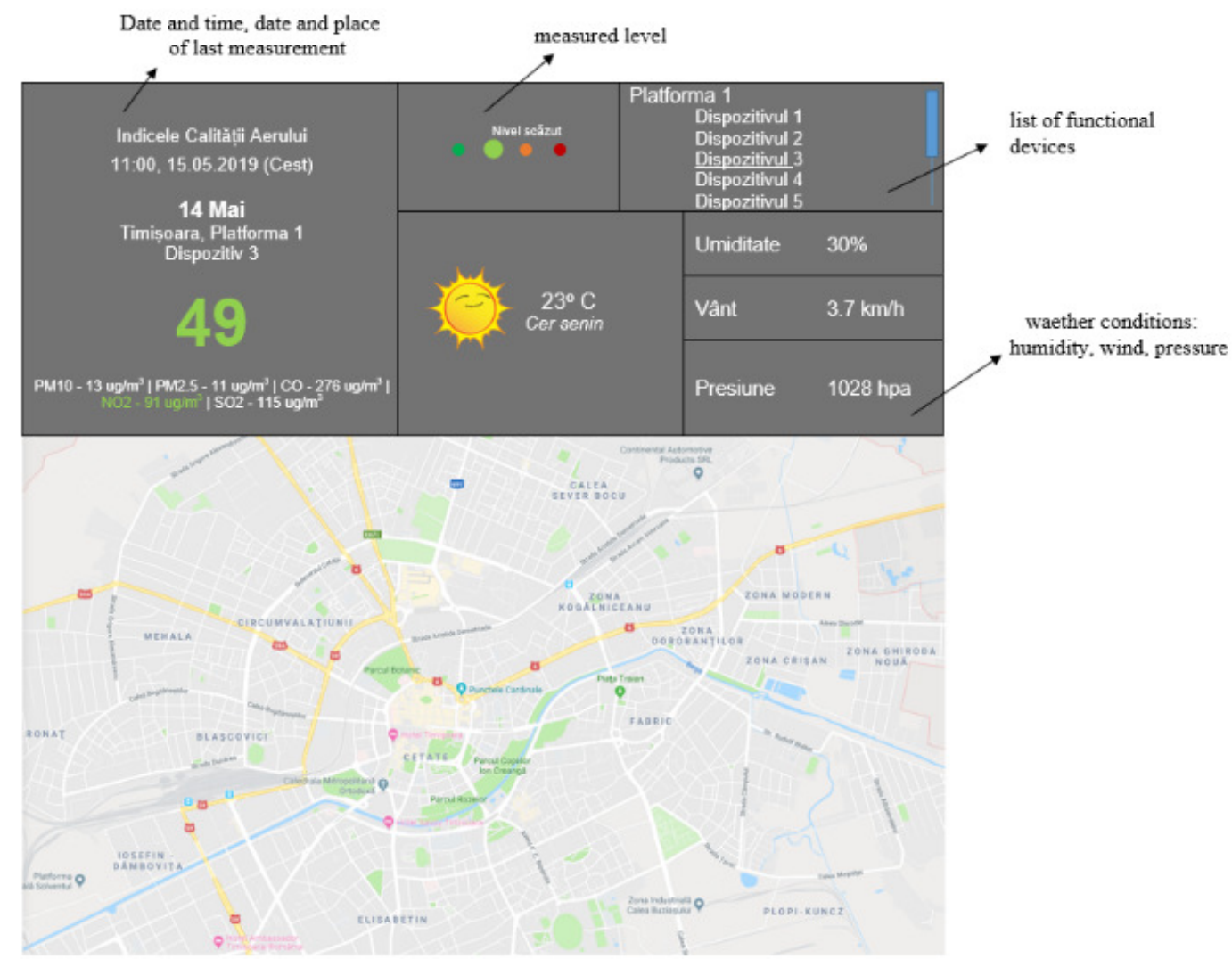

Figure 5: Graphical user interface - online platform

\section{Results and discussions}

The purpose of this paper is not the analysis of the air quality in an area of interest but the presentation of a low-cost (table 1) air quality monitoring device that provides correct data.

The data collected by the sensors are sent to a database from where they are displayed by the online platform such that to be easy to understand for the end user.

In the online platform, the user can select the period for which he wants to display the results. The results are displayed as graphs. Parameter values for one hour or several hours can be displayed (figures 6, 7, 8), for a week, a month or for a certain peri-od (figures 9, 10 for the 01.10.2019 08.10.2019 period). 


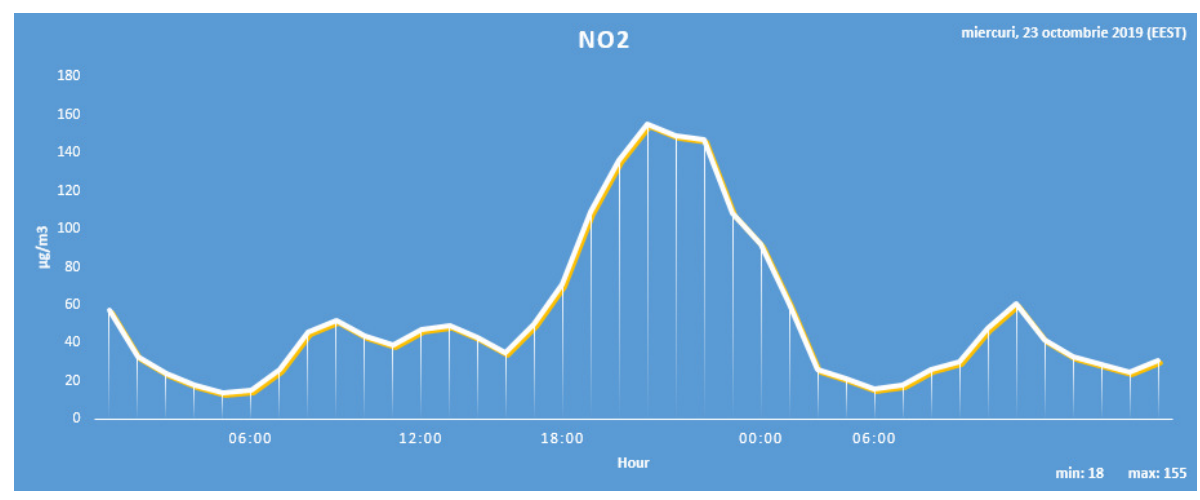

Figure 6: NO2 values for 24h (22.10.2019 - 23.10.2019)

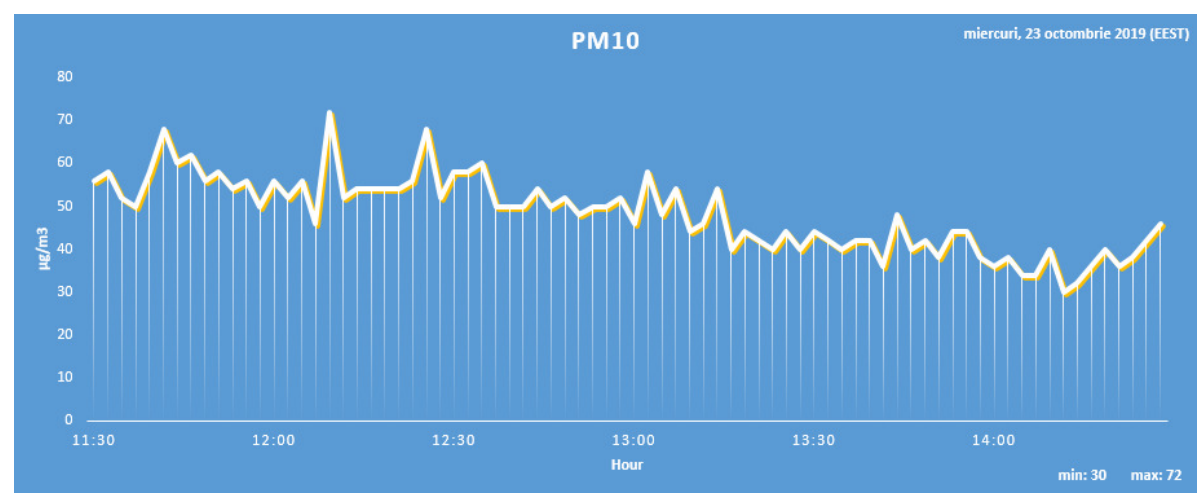

Figure 7: PM10 values for 3h (23.10.2019)

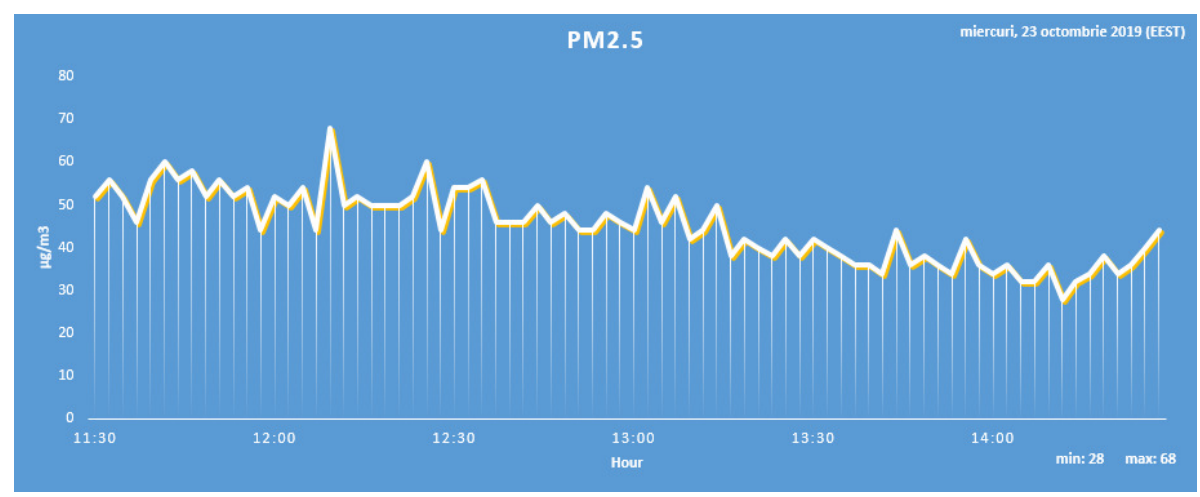

Figure 8: PM2.5 values for 3h (23.10.2019)

All the parameters discussed in this paper can be displayed in this form (PM10, PM2.5, SO2, $\mathrm{NO} 2, \mathrm{CO}, \mathrm{O} 3, \mathrm{VOC}, \mathrm{CO} 2)$.

After 7 months of operation in real conditions and with repeated comparisons between the data collected by these devices and data from parallel measurements with government operated air quality stations, it can be concluded that the devices provide reliable values (figures 11, 12, 13).

After mounting all devices, certain problems appeared, related to the locations select-ed initially. Due to poor air flow the results were not relevant. In the first phase, these devices were relocated, and in the second phase the number of holes on the side of these devices was increased. The measured data clearly show that in certain periods of time the values of the air quality parameters do not comply with legal norms and concrete measures must be taken. 


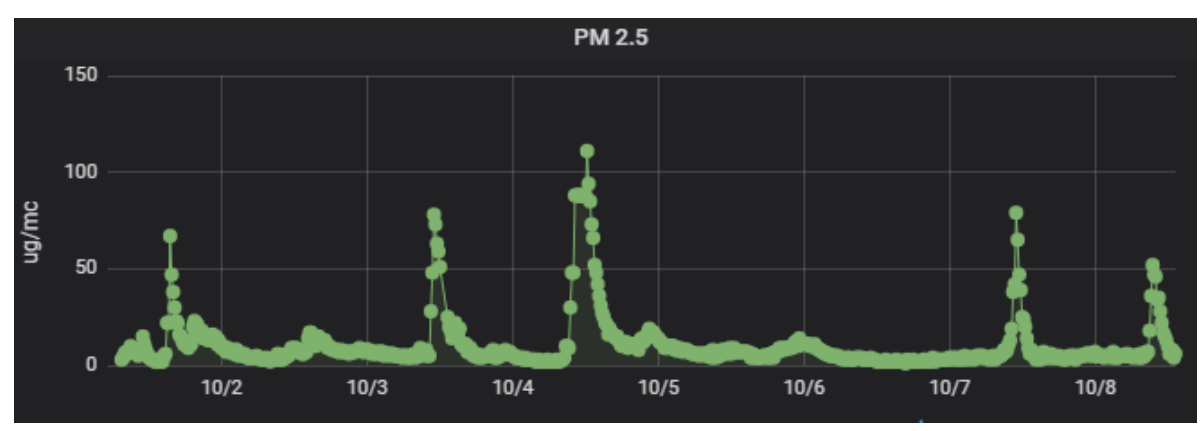

Figure 9: PM2.5 values for 1 week (01.10.2019 - 08.10.2019)

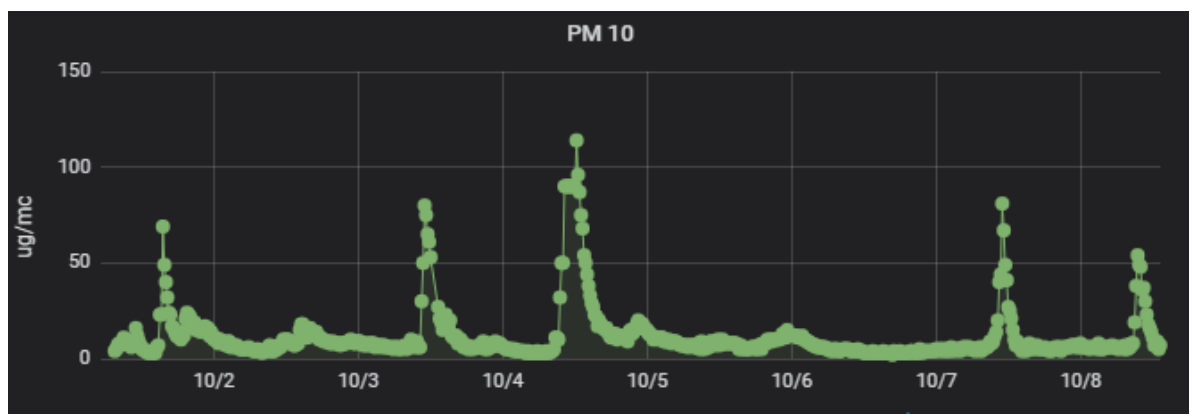

Figure 10: PM10 values for 1 week (01.10.2019 - 08.10.2019)

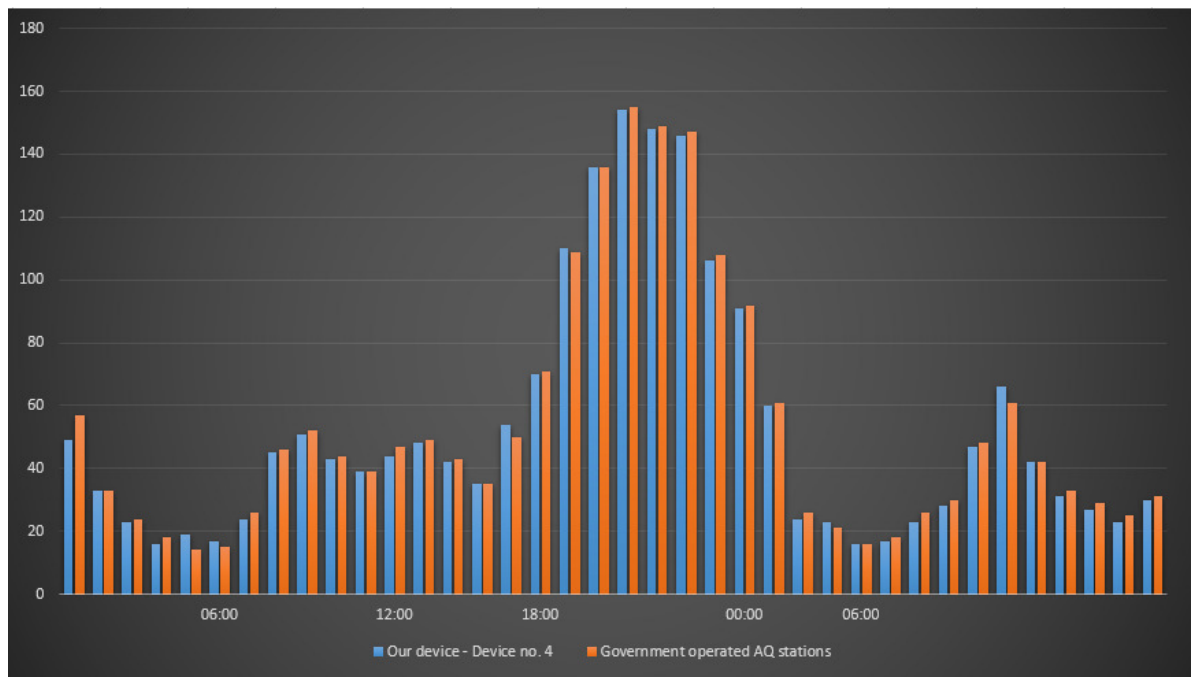

Figure 11: Comparison between governments operated air quality station data and our low-cost devices data - NO2 values for 24h (22.10.2019 - 23.10.2019) 


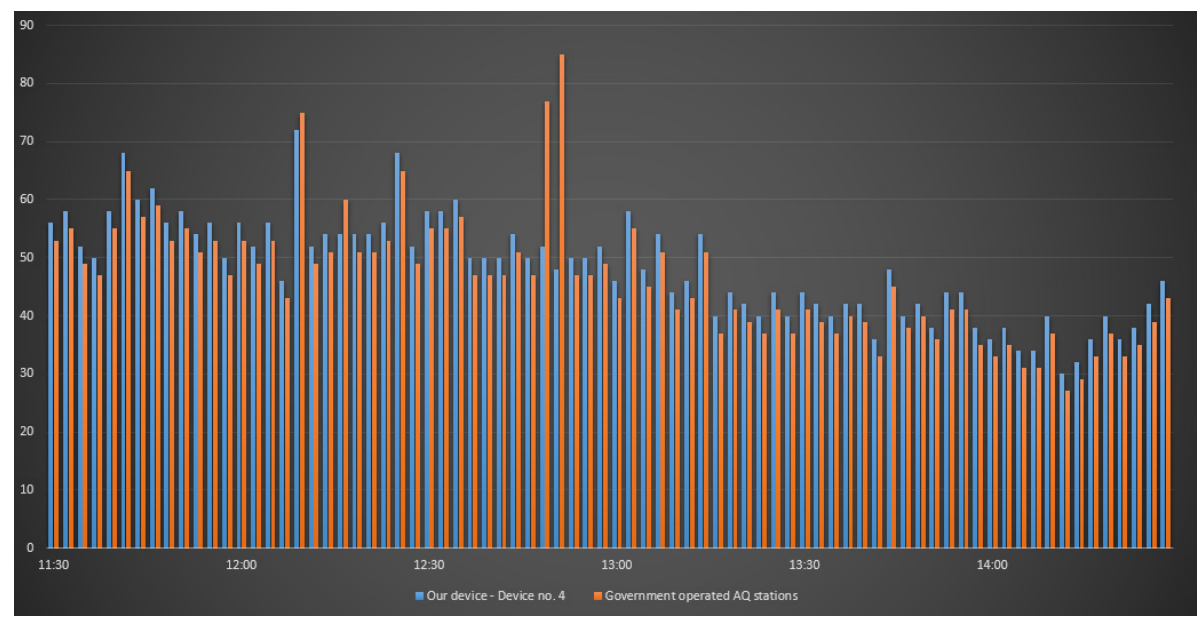

Figure 12: Comparison between governments operated air quality station data and our low-cost devices data - PM10 values for 3h (23.10.2019)

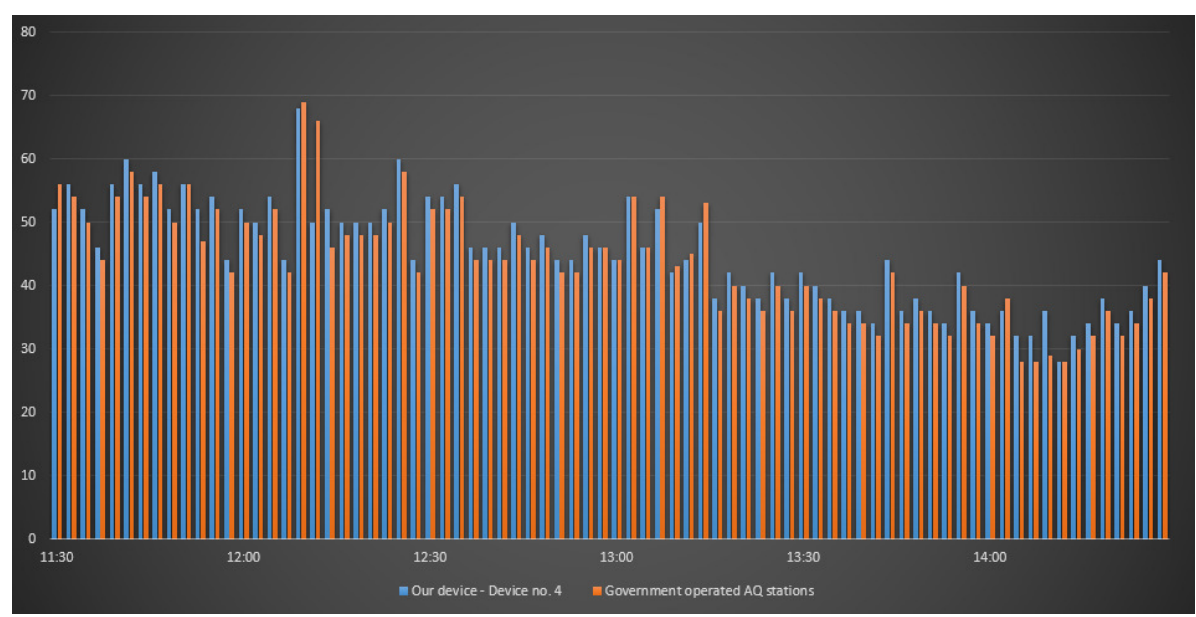

Figure 13: Comparison between governments operated air quality station data and our low-cost devices data - PM2.5 values for 3h (23.10.2019) 


\section{Conclusions}

Due to the dynamic development of the city of Timisoara, in many areas, air quality problems arise (chaotic development on the real estate market, due to which the industrial platforms are located either very close to the residential areas or already found inside the city). Also, the car traffic is increasing near the industrial areas. Plus, any method is welcome to follow the air quality in the industrial platforms regarding the change of the filters in the exhaust systems.

There are many established methods, but we wanted to meet the needs of the economic environment with a low-cost device, which can provide the same information and quality. More than necessary, our devices from this pilot project, can send data to the application server through LoRaWAN, without having to build a complex and expensive network such as a Wi-Fi network.

After this pilot project implementation, we can conclude that this air quality monitoring device can operate at a city scale, due to the implementation of LoRaWAN as communication protocol.

Further works will focus on increasing the autonomy of the devices, so that where they will need to be mounted in places where there is no electricity supply. The existing model has autonomy only 2-3 days depending on the number of readings per day.

\section{References}

[1] Arroyo, P.; Herrero, J.L.; Suárez, J.I.; Lozano, J. (2019). Wireless Sensor Network Combined with Cloud Computing for Air Quality Monitoring, Sensors, 19(3), 1-17, 2019.

[2] Borrego, C.; Costa, A.M.; Ginja, J.; Amorim, M.et al. (2016). Assessment of air quality microsensors versus reference methods: The EuNetAir joint exercise, Atmospheric Environment, 147, 246-263, 2016.

[3] Castell, N.; Dauge, F.R.; Schneider, F. et al. (2017). Can commercial low-cost sensor platforms contribute to air quality monitoring and exposure estimates?, Environment International, 99, 293-302, 2017.

[4] Hasenfratz, D.; Saukh, O.; Walser, C. et al. (2016). Deriving high-resolution urban air pollution maps using mobile sensor nodes, Pervasive and Mobile Computing, 16(B), 268-285, 2016.

[5] Honeywell (2018). HPM Series Particulate Matter Sensors, Honeywell, 2018.

[6] Johnston, S.J.; Basford, P.J.; Bulot, F.M.J. et al. (2019). City Scale Particulate Matter Monitoring Using LoRaWAN Based Air Quality IoT Devices, Sensors, 19(1), 1-20, 2019

[7] Lee, S.; Lim, S. B. (2015). Applications of Ubiquitous Sensor Network: Micro-Scale Air Quality Monitoring, International Journal of Computers Communications \& Control, 10(3), 370-381, 2015 .

[8] Mo, X.; Zhang, L.; Li, H.; Qu, Z. (2019). A Novel Air Quality Early-Warning System Based on Artificial Intelligence, Environmental Research and Public Health, 16(19), 1-25, 2019.

[9] Oprea, M.; Mihalache, S. F.; Popescu, M. (2017). Computational Intelligence-based PM2.5 Air Pollution Forecasting, International Journal of Computers Communications \& Control , 12(3), 365-380, 2017.

[10] Simo, A.; Barbulescu, C.; Salinschi, M. (2018). LoRaWAN based industrial emission monitoring with self-flying drone, In 18th International Multidisciplinary Scientific GeoConference, 503-510, 2018 .

[11] Wang, S.Y.; Zou J.J.; Chen Y.R. et al. (2018). Long-Term Performance Studies of a LoRaWANBased PM2.5 Application on Campus, In 87th Vehicular Technology Conference (VTC Spring), $1-6,2018$. 
[12] Wesseling, J.; de Ruiter, H.; Blokhuis, C. et al.(2019). Development and Implementation of a Platform for Public Information on Air Quality, Sensor Measurements, and Citizen Science, Atmosphere, 10(8), 1-20, 2019.

\section{(c) (i) (9)}

Copyright (C) 2020 by the authors. Licensee Agora University, Oradea, Romania.

This is an open access article distributed under the terms and conditions of the Creative Commons Attribution-NonCommercial 4.0 International License.

Journal's webpage: http://univagora.ro/jour/index.php/ijccc/

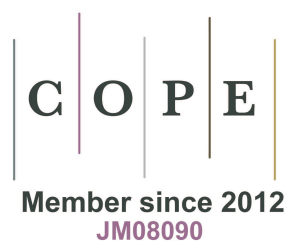

This journal is a member of, and subscribes to the principles of, the Committee on Publication Ethics (COPE).

https://publicationethics.org/members/international-journal-computers-communications-and-control

Cite this paper as:

Simo, A.; Dzitac, S.; Frigura-Iliasa, F. M.; Musuroi, S.; Andea, P.; Meianu, D. (2020). Technical Solution for a Real-Time Air Quality Monitoring System, International Journal of Computers Communications \& Control, 15(4), 3891, 2020. https://doi.org/10.15837/ijccc.2020.4.3891 